\title{
Mitochondrial SNP markers to monitor evolutionary lineage ancestry in Apis mellifera mellifera conservation programs
}

\author{
Dora Henriques ${ }^{1}$, Melanie PARejo ${ }^{2,3}$, Ana R. Lopes ${ }^{1}$, Maria Alice Pinto ${ }^{1}$ \\ ${ }^{1}$ Centro de Investigação de Montanha, Instituto Politécnico de Bragança, Campus de Santa Apolónia, 5300-253, \\ Bragança, Portugal \\ ${ }^{2}$ Agroscope, Swiss Bee Research Centre, Bern, Switzerland \\ ${ }^{3}$ Department of Genetics, Physical Anthropology and Animal Physiology University of the Basque Country (UPV/ \\ EHU), Leioa, Spain
}

Received 20 February 2019 - Revised 17 May 2019 - Accepted 13 June 2019

\section{Apis mellifera mellifera / mtDNA / maternal lineage / SNPs / conservation}

The European dark honey bee, Apis mellifera mellifera, is threatened in most of its native range, in part, due to introgressive hybridization with bees from the highly divergent $\mathrm{C}$-lineage, mainly Apis mellifera carnica and Apis mellifera ligustica (De la Rúa et al. 2009; Pinto et al. 2014). Yet, the maintenance of locally adapted genetic diversity is critical for the population long-term survival and sustainability (De la Rúa et al. 2009; Meixner 2010). The growing awareness that genetic diversity is important for sustainable beekeeping led to implementation of different conservation and breeding programs throughout Europe, which are in need of reliable and costefficient molecular tools to accurately monitor Clineage introgression into A. m. mellifera (De la

Electronic supplementary material The online version of this article (https://doi.org/10.1007/s13592-019-00666-z) contains supplementary material, which is available to authorized users.

Corresponding author: M. Pinto, apinto@ipb.ptA. Pinto, apinto@ipb.pt

Dora Henriques and Melanie Parejo contributed equally to this work.

Manuscript editor: Marina Meixner
Rúa et al. 2009; Henriques et al. 2018a, b; Meixner 2010). The large mating flight distances and the polyandrous mating system make it challenging to preserve honey bee subspecies in an open conservation area where intruders can fly in (Neumann et al. 1999). It is therefore necessary to regularly control the genetic ancestry of new or superseded colonies.

A number of studies have designed diagnostic tools with nuclear (nc) SNP markers capable of discriminating different honey bee lineages (Chapman et al. 2015, 2017; Muñoz et al. 2015; Parejo et al. 2016). Recently, a set of highly informative ncSNPs for estimating C-derived introgression into A. m. mellifera (Muñoz et al. 2015) were combined into four assays, which were tested and validated for genotyping in the Agena BioScience ${ }^{\text {TM }}$ iPLEX MassARRAY platform (Henriques et al. 2018a). The number of ncSNPs included in each of the four assays $(\mathrm{M} 1=34, \mathrm{M} 2=32, \mathrm{M} 3=28, \mathrm{M} 4=23)$ is lower than the maximum capacity of the iPLEX protocol (40 SNPs) allowing additional markers, such as mitochondrial SNPs (mtSNPs). By combining SNPs of both mitochondrial and nuclear 
compartments, a more complete identification is achieved in a single genotyping step.

Analyzing mtDNA, in addition to ncDNA, enables better-informed decision-making in $A$. $m$. mellifera conservation and breeding programs. Due to independent transmission of nuclear and mitochondrial DNA, a colony carrying C-derived mtDNA can be identified as pure $A$. $m$. mellifera at the nuclear level, after several generations of backcrossing with $A$. $m$. mellifera drones (Jensen et al. 2005; Meixner et al. 2013). Then, whether a colony exhibiting cytonuclear incongruence is maintained in a conservation area is a decision to be made by the conservation manager.

The aim of this study was to improve the SNPs assays developed by Henriques et al. (2018a) adding informative mitochondrial SNPs. To this end, we used whole mtDNA sequence data of 155 drones, each representing a single colony, collected across Europe (Table S1) (Henriques et al. 2019; Parejo et al. 2016). Mapping and variant calling were performed following best practices detailed in Henriques et al. (2018b) (see Table S1 for further details). A total of 397 SNPs were identified in the 16,343 bp reference mitochondrial genome (Crozier and Crozier 1993). These were distributed across the 13 protein-coding genes with ATP8 containing the lowest (5) and COX1 the highest number (46) of SNPs (Table S2). Maternal ancestry of the 155 individuals was assessed by a neighbor-joining (NJ) tree constructed in MEGA7 (Tamura et al. 2013). A total of 104 individuals were placed in the Mlineage cluster and 51 in the C-lineage cluster (Table S1; Fig. S1).

To select informative mtDNA markers, we randomly chose $78 \mathrm{M}$ - and $38 \mathrm{C}$-lineage individuals as training set and $26 \mathrm{M}$ - and $13 \mathrm{C}$-lineage as holdout set (following Anderson (2010)). $F_{\text {ST }}$ values between $\mathrm{M}$ - and $\mathrm{C}$-derived individuals were inferred for each mtSNP from the training set using PLINK 1.9 (Chang et al. 2015), and those showing $F_{\text {ST }}$ values $>0.75$ were selected. Sequence information of the flanking regions (250 bp) of the selected mtSNPs was then used to supplement the four ncSNP assays (Henriques et al. 2018a) using the Replex option of the software AssayDesign 4.0 (Agena BioScience ${ }^{\mathrm{TM}}$ ). The software searched for optimal areas within the flanking regions to design forward, reverse, and iPLEX extension primers compatible with those published in Henriques et al. (2018a).

From a total of $397 \mathrm{mtSNPs}, 193$ were highly informative $\left(F_{\mathrm{ST}}>0.75\right.$, Table S2). Of these, the assay design tool was able to incorporate five into the M4 ncSNP assay. The five mtSNPs are located in COX1, ND4, and ls-rRNA (Table I). To evaluate their power for distinguishing $\mathrm{M}$ - and $\mathrm{C}$-lineage colonies, a NJ tree was constructed using the holdout data set. The NJ tree shows that the five mtSNPs are sufficient to discriminate colonies of C- from M-lineage maternal ancestry (Fig. S2).

The five informative mtSNPs could only be added to the M4 ncSNP assay designed previously (Henriques et al. 2018a), which contained the lowest number of ncSNPs (23). Henriques et al. (2018a) showed that assay M1 (34 ncSNPs) has the best individual performance in estimating nuclear C-lineage introgression. Accordingly, a good approach for concurrent inference of nuclear and mitochondrial ancestry could be to combine M1 with the new 28-plex M4 (23 ncSNPs plus $5 \mathrm{mtSNPs}$ ). We therefore tested if the introgression proportions ( $Q$ values) estimated from the ncSNP assays M1 + M4 were similar to those obtained from the fourassays together(Henriques et al. 2018a). From the 117 SNPs included in the four assays, we were able to extract 113 from the 155 whole-genome (WG) sequences. Werun ADMIXTURE (Alexander et al. 2009) in the 155 individuals using the four assays combined (113 ncSNPs) and M1 + M4 (55 ncSNPs, as 2 of them were not in the WG sequences). $Q$ values were estimated for $K=2$ using 10,000 iterations in 20 independent runs. $Q$ values estimated with $\mathrm{M} 1+$ M4 were highly correlated with those estimated with the 4 assays $(r=0.995, P$ value $<0.001)$, and the differences between them were negligible (mean absolute difference $=0.027$; Table S3) .

There is a need of accurate identification of C-lineage introgressed colonies for an efficient management of $A$. m. mellifera conservation programs. A more complete identification requires informative markers of both nuclear and mitochondrial compartments (Meixner et al. 2013). For example, in this study, cytonuclear incongruence is observed in two A. m. ligustica (C-lineage) individuals that carry mitochondria 
Table I. Genomic information of the five selected SNPs to distinguish C and M maternal lineages

\begin{tabular}{lcllcc}
\hline Locus name & Position & Gene & Annotation & $F_{\text {ST }}$ (total*) & $F_{\text {ST }}$ (training*) \\
\hline MT_2447_CT & 2447 & COX1 & Synonymous & 1.000 & 1.000 \\
MT_2687_TC & 2687 & COX1 & Synonymous & 1.000 & 1.000 \\
MT_13513_GA & 13513 & ls-rRNA & - & 0.732 & 0.764 \\
MT_9361_CT & 9361 & ND4 & Nonsynonymous & 0.987 & 0.982 \\
MT_2939_TA & 2939 & COX1 & Synonymous & 1.000 & 1.000 \\
\hline
\end{tabular}

*Estimated using the total and training sets

of M-lineage ancestry (Table S3). Here, we improved the recently designed M4 ncSNP assay (Henriques et al. 2018a) by adding five mtSNPs and provided PCR and iPLEX primers for genotyping in the MassARRAY platform (Table S4). We showed that C-lineage introgression into $A$. $m$. mellifera can be accurately estimated by combining the new cytonuclear M4 assay improved here with the best performing M1 nuclear assay developed previously (Henriques et al. 2018a). The combined M1 + M4 assays, containing 57 nuclear and five mitochondrial SNPs, provide a robust molecular tool for assisting management decisions toward protection and reestablishment of the endangered A. m. mellifera .

\section{ACKNOWLEDGMENTS}

We thank João Costa (Instituto Gulbenkian de Ciência, Oeiras, Portugal) for redesigning the M4 assay. Analyses were performed using computational resources at the Instituto Politécnico de Bragança.

Les marqueurs SNP mitochondriaux pour surveiller l'ascendance de la lignée évolutionnaire dans les programmes de conservation de Apis mellifera mellifera

Wissenschaftliche Notiz: Mitochondriale SNP Marker zur Überwachung der evolutionären Herkunftslinie in Erhaltungsprogrammen von Apis mellifera mellifera

\section{AUTHOR CONTRIBUTIONS}

MAP and DH conceived the ideas and designed the methodology. DH and MP performed most of the analyses with assistance of ARL. MP and DH wrote the manuscript with input from MAP. All the authors critically reviewed the manuscript for important intellectual content.

\section{FUNDING INFORMATION}

This work was financed by FEDER (Fundo Europeu de Desenvolvimento Regional) through the program COMPETE 2020-POCI (Programa Operacional para a Competitividade e Internacionalização) and by Portuguese funds through FCT (Fundação para a Ciência e a Tecnologia) in the framework of the project BeeHappy (POCI-01-0145-FEDER-029871). Melanie Parejo was supported by a mobility fellowship awarded from the Swiss National Science Foundation (SNSF).

\section{COMPLIANCE WITH ETHICAL STANDARDS}

Conflict of interest The authors declare that they have no conflict of interest.

\section{REFERENCES}

Alexander, D. H., Novembre, J., Lange, K. (2009) Fast model-based estimation of ancestry in unrelated individuals Genome research 19:1655-1664 
Anderson, E. (2010) Assessing the power of informative subsets of loci for population assignment: standard methods are upwardly biased Molecular Ecology Resources 10:701-710

Chang, C. C., Chow, C. C., Tellier, L. C., Vattikuti, S., Purcell, S. M., Lee, J. J. (2015) Second-generation PLINK: rising to the challenge of larger and richer datasets Gigascience 4:7

Chapman, N. C., Harpur, B. A., Lim, J., Rinderer, T. E., Allsopp, M. H., Zayed, A., Oldroyd, B. P. (2015) A SNP test to identify Africanized honeybees via proportion of 'African'ancestry Molecular Ecology Resources 15:1346-1355

Chapman, N. C., Bourgeois, A. L., Beaman, L. D., Lim, J., Harpur, B. A., Zayed, A., Allsopp, M. H., Rinderer, T. E., Oldroyd, B. P. (2017) An abbreviated SNP panel for ancestry assignment of honeybees (Apis mellifera) Apidologie 48:776-783

Crozier, R., Crozier, Y. (1993) The mitochondrial genome of the honeybee Apis mellifera: complete sequence and genome organization Genetics 133:97-117

De la Rúa, P., Jaffé, R., Dall'Olio, R., Muñoz, I., Serrano, J. (2009) Biodiversity, conservation and current threats to European honeybees Apidologie 40:263-284

Henriques, D., Browne, K. A., Barnett, M. W., Parejo, M., Kryger, P. et al. (2018a) High sample throughput genotyping for estimating $\mathrm{C}$-lineage introgression in the dark honeybee: an accurate and cost-effective SNPbased tool Scientific Reports 8:8552

Henriques, D., Parejo, M., Vignal, A., Wragg, D., Wallberg, A., Webster, M. T., Pinto, M. A. (2018b). Developing reduced SNP assays from wholegenome sequence data to estimate introgression in an organism with complex genetic patterns, the Iberian honeybee (Apis mellifera iberiensis). Evolutionary applications, 11(8), 12701282.

Henriques, D., Chávez-Galarza, J., Quaresma, A., Neves, C. J., Lopes, A. R., Costa, C., Costa, F. O., Rufino, J., Pinto, M. A. (2019) From the popular tRNA leuCOX2 intergenic region to the mitogenome: insights from diverse honey bee populations of Europe and North Africa. Apidologie, 50(2), 215-229.
Jensen, A. B., Palmer, K. A., Boomsma, J. J., Pedersen, B. V. (2005) Varying degrees of Apis mellifera ligustica introgression in protected populations of the black honeybee, Apis mellifera mellifera, in northwest Europe Molecular Ecology 14:93-106

Meixner, M. D. (2010) A historical review of managed honey bee populations in Europe and the United States and the factors that may affect them Journal of Invertebrate Pathology 103:S80-S95

Meixner, M. D., Pinto, M. A., Bouga, M., Kryger, P., Ivanova, E., Fuchs, S. (2013) Standard methods for characterising subspecies and ecotypes of Apis mellifera Journal of Apicultural Research 52:1-28

Muñoz, I., Henriques, D., Johnston, J. S., Chávez-Galarza, J., Kryger, P., Pinto, M. A. (2015) Reduced SNP panels for genetic identification and introgression analysis in the dark honey bee (Apis mellifera mellifera) PloS ONE 10:e0124365

Neumann, P., Moritz, R. F., van Praagh, J. (1999) Queen mating frequency in different types of honey bee mating apiaries Journal of Apicultural Research 38:11-18

Parejo, M., Wragg, D., Gauthier, L., Vignal, A., Neumann, P., Neuditschko, M. (2016) Using whole-genome sequence information to foster conservation efforts for the European dark honey bee, Apis mellifera mellifera Frontiers in Ecology and Evolution 4:140

Pinto, M. A., Henriques, D., Chávez-Galarza, J., Kryger, P., Garnery, L. et al. (2014) Genetic integrity of the Dark European honey bee (Apis mellifera mellifera) from protected populations: a genome-wide assessment using SNPs and mtDNA sequence data Journal of Apicultural Research 53:269-278 doi:https://doi. org/10.3896/IBRA.1.53.2.08

Tamura, K., Stecher, G., Peterson, D., Filipski, A., Kumar, S. (2013) MEGA6: molecular evolutionary genetics analysis version 6.0 Molecular Biology and Evolution 30:2725-2729

Publisher's note Springer Nature remains neutral with regard to jurisdictional claims in published maps and institutional affiliations. 